\title{
Correction to: Self-injective Cellular Algebras Whose Representation Type are Tame of Polynomial Growth
}

\section{S. Ariki ${ }^{1} \cdot$ R. Kase ${ }^{2} \cdot$ K. Miyamoto ${ }^{1} \cdot$ K. Wada ${ }^{3}$}

Published online: 4 May 2019

(C) Springer Nature B.V. 2019

\section{Erratum to: Algebras and Representation Theory https://doi.org/10.1007/s10468-019-09872-w}

The original version of this article unfortunately contains mistakes introduced during the production phase. The mistakes are on the incorrect cross-referencing of Theorem 6.8, Theorem 7.1, Theorem 7.16, Theorem 7.20, Proposition 5.4, Proposition 5.5, Proposition 6.2, Proposition 6.4, Proposition 6.5, Proposition 6.6, Proposition 6.7, Proposition 7.2, Proposition 7.4, Proposition 7.7, Proposition 7.9, Proposition 7.12, Proposition 7.14, Proposition 7.19, Lemma 5.3, Lemma 6.3, Lemma 7.6, Lemma 7.17, Lemma 7.18, Definition 7.3, Definition 7.5, Definition 7.8, Definition 7.10, Definition 7.13, Definition 7.15 found in sections $1,5,6,7,8$. This is due to the change in the numbering of subsections $5.1,6.1,6.2,6.3,6.4$, 7.1, 7.2, 7.3, and 7.4.

The original article has been corrected.

The online version of the original article can be found at https://doi.org/10.1007/s10468-019-09872-w.

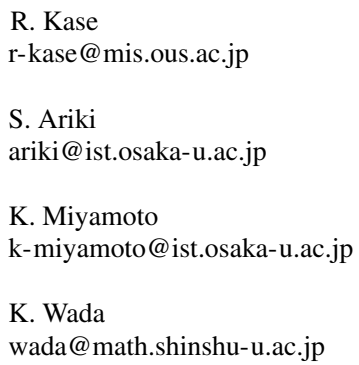

1 Department of Pure and Applied Mathematics, Graduate School of Information Science and Technology, Osaka University, 1-5 Yamadaoka, Suita, Osaka 565-0871, Japan

2 Faculty of Informatics, Okayama University of Science, 1-1 Ridaicho, Kita-ku, Okayama-shi, 700-0005, Japan

3 Department of Mathematics, Faculty of Science, Shinshu University, Asahi 3-1-1, Matsumoto, 390-8621, Japan 
Publisher's Note Springer Nature remains neutral with regard to jurisdictional claims in published maps and institutional affiliations. 